\title{
Fixed Point Theorems in Complex Valued Quasi b-Metric Spaces for Satisfying Rational Type Contraction
}

\author{
J. Uma Maheswari, A. Anbarasan", M. Ravichandran \\ Department of Mathematics, St. Joseph's College (Autonomous), Tiruchirappalli - 620 002, India
}

Received November 26, 2020; Revised January 29, 2021; Accepted February 17, 2021

\section{Cite This Paper in the following Citation Styles}

(a): [1] J. Uma Maheswari, A. Anbarasan, M. Ravichandran, "Fixed Point Theorems in Complex Valued Quasi b-Metric Spaces for Satisfying Rational Type Contraction," Mathematics and Statistics, Vol. 9, No. 1, pp. 59 - 64, 2021. DOI: 10.13189/ms.2021.090110.

(b): J. Uma Maheswari, A. Anbarasan, M. Ravichandran (2021). Fixed Point Theorems in Complex Valued Quasi b-Metric Spaces for Satisfying Rational Type Contraction. Mathematics and Statistics, 9(1), 59 - 64. DOI: 10.13189/ms.2021.090110.

Copyright $\mathrm{C} 2021$ by authors, all rights reserved. Authors agree that this article remains permanently open access under the terms of the Creative Commons Attribution License 4.0 International License

\begin{abstract}
The notion of complex valued metric spaces proved the common fixed point theorem that satisfies rational mapping of contraction. In the contraction mapping theory, several researchers demonstrated many fixed-point theorems, common fixed-point theorems and coupled fixed-point theorems by using complex valued metric spaces. The idea of $b$-metric spaces proved the fixed point theorem by the principle of contraction mapping. The notion of complex valued b-metric spaces, and this metric space was the generalization of complex valued metric spaces. They explained the fixed point theorem by using the rational contraction. In the metric spaces, we refer to this metric space as a quasi-metric space, the symmetric condition $\mathrm{d}(\mathrm{x}, \mathrm{y})=\mathrm{d}(\mathrm{y}, \mathrm{x})$ is ignored. Metric space is a special kind of space that is quasi-metric. The Quasi metric spaces were discussed by many researchers. Banach introduced the theory of contraction mapping and proved the theorem of fixed points in metric spaces. We are now introducing the new notion of complex quasi b-metric spaces involving rational type contraction which proved the unique fixed point theorems with continuous as well as non-continuous functions. Illustrate this with example.
\end{abstract}

2010 Mathematical subject classification: $46 \mathrm{~S} 40,47 \mathrm{H} 10$, $54 \mathrm{H} 25$

Keywords Fixed Point, Metric Space, Quasi Metric Space, Complex Valued Metric Space, Complex Valued Quasi b-metric Space

\section{Introduction}

The complex value metric spaces were first introduced by Azam, et.al [1] and rational contraction mappings were used to prove the theorems. Czerwik introduced b-metric spaces, which is the generalization of metric spaces [2]. The complex valued b-metric spaces were introduced by Rao et al. [3] and so many researchers demonstrated the complex valued b-metric spaces.

See [4] in 2012, the good results on Nonlinear contractions in partially ordered quasi b-metric spaces were proved by H. Shah and N. Hussian. The metric fixed point theorems were generated by Banach [5] in 1922. By means of rational expression, P.Dhivya and M.Marudai [6] demonstrated the theorem of complex partial metric spaces. By using rational expression, M. Imdad, T. I. Khan [7] proved non-continuous mappings of fixed point theorem. and J. Uma Maheswari and A Anbarasan [8] were involved in generalizing the contraction mapping principle to prove very good results in the theory of fixed points.

In complex valued metric spaces, many ways of generalization of metric spaces are proven.[9] F.Rouzkard and M.Imdad have demonstrated some common fixed point theorems on complex metric space and common fixed point mappings that meet rational inequalities in 
ordered complex generalized metric spaces by [10] Abbas, M, Cajbaic Rajic V, Nazir.T and Radenovic.

In complex valued b-metric spaces, several fixed point theorems have been demonstrated by [11] A.k Dubey, Rita Shukla and R.P. Dubey. A new definition of complex valued quasi b-metric spaces satisfying rational form contraction is now interested in proving fixed point theorems.

Now,

We give definitions which are beneficial of our main results.

Consider that $\mathbb{C}$ represents the complex numbers and $q_{1}, q_{2} \in \mathbb{C}$. Let us define a partial order $\leq$ on $\mathbb{C}$ as pursue:

$$
\begin{aligned}
& q_{1} \leq q_{2} \text { if and only if R.p.rt }\left(q_{1}\right) \leq \operatorname{R} \cdot \operatorname{prrt}\left(q_{2}\right), \\
& I y \cdot p \cdot r t\left(q_{2}\right) \leq I y \cdot \operatorname{prt}\left(q_{2}\right) .
\end{aligned}
$$

It pursues that $q_{1} \leq q_{2}$ if any one of the following conditions is satisfied:

(i) R.p.rt $\left(q_{1}\right)=R \cdot p \cdot r t\left(q_{2}\right)$,

Iy.p.rt $\left(q_{1}\right)<I y \cdot p \cdot r t\left(q_{2}\right)$,

(ii) R.p.rt $\left(q_{1}\right)<R \cdot p \cdot r t\left(q_{2}\right)$,

Iy.p.rt $\left(q_{1}\right)=I y \cdot p \cdot r t\left(q_{2}\right)$,

(iii) R.p.rt $\left(q_{1}\right)<R \cdot p \cdot r t\left(q_{2}\right)$,

Iy.p.rt $\left(q_{1}\right)<\operatorname{Iy} \cdot p \cdot r t\left(q_{2}\right)$,

(iv) R.p.rt $\left(q_{1}\right)=R \cdot p \cdot r t\left(q_{2}\right)$,

$I y \cdot p \cdot r t\left(q_{1}\right)=I y \cdot p \cdot r t\left(q_{2}\right)$,

In particular, we write $q_{1}<q_{2}$ if $q_{1} \neq q_{2}$ and any one of this (i), (ii) and (iv) is satisfied and we write $q_{1}<q_{2}$ if only (iii) is satisfied.

\section{Definition 1.1}

A complex quasi metric on a non-void set $\mathrm{L}$ is a mapping $p_{c q b}: L \times L \rightarrow C$ such that for each $a, b, e \varepsilon L$.

(1) $p_{c q b}(a, b)=0$ if and only if $a=b$.

(2) $p_{c q b}(a, b) \leq p_{c q b}(a, e)+p_{c q b}(e, b)$.

\section{Definition1.2}

Consider $s \geq 1$ be a real number. A non- void set $\mathrm{L}$ is a function $p_{c q b}: L \times L \rightarrow C$ is known as complex valued quasi $\mathrm{b}$ metric spaces if for every $a, b, e \in L$ :

$$
\text { (1) } p_{c q b}(a, b)=0 \text { iff } a=b
$$

(2) $p_{c q b}(a, b) \leq s\left[p_{c q b}(a, e)+p_{c q b}(e, b)\right]$

\section{Definition 1.3}

A non-void set $\mathrm{L}$ is a mapping $p_{c q b}: L \times L \rightarrow C$ is known as complex valued quasi metric space and as well as complex valued quasi b-metric spaces.

1) A sequence $\left\{b_{m}\right\}$ is said to be converges to an element $b \in L$ if given $k>0$ and $k \in C$ there exists a natural number $\mathrm{n}$ such that $p_{c q b}\left(b_{m}, b\right)<k$ for every $m \geq n$.

2) A sequence $\left\{b_{m}\right\}$ is said to be Cauchy sequence if there is $u \in C$ given $k>0$ there exist a positive integer $n_{0}$ such that $\left|p_{c q b}\left(b_{m}, b_{n}\right)-u\right|<k$ for each $m, n \geq n_{0}$

3) Let $\left(L, p_{c q b}\right)$ is said to be complete if for all Cauchy sequence $\left\{b_{m}\right\}$ of $\mathrm{L}$ which converges to an element of $\mathrm{L}$.

\section{Main Result}

First we prove the unique fixed point theorem using complex quasi metric spaces.

\section{Theorem 2.1.}

Let $\left(L, p_{c q b}\right)$ be a quasi metric space. Suppose that there exists a complex quasi metric space $p_{c q b}$ on $\mathrm{L}$ such that $\left(L, p_{c q b}\right)$ is a complete complex quasi metric space. Let $F: L \rightarrow L$ be a self mapping and continuous. There exist positive real numbers $\lambda, \mu, \delta$ with

$$
\begin{gathered}
\lambda+2 \delta<1 p_{c q b}(F a, F b) \leq \lambda p_{c q b}(a, b)+ \\
\mu \frac{p_{c q b}(a, F a) p_{c q b}(b, F a)}{1+p_{c q b}(a, b)}+ \\
\delta \frac{\left[p_{c q b}(F a, b)+p_{c q b}(a, b)\right]\left[p_{c q b}(a, F b)\right]}{p_{c q b}(a, b)}
\end{gathered}
$$

Then $\mathrm{F}$ has a unique fixed point.

\section{Proof.}

First we prove that $\mathrm{F}$ has a fixed point.

Let us take $a_{0} \varepsilon L$

Define a $F a_{2 n}=a_{2 n+1}$ and $F a_{2 n+1}=a_{2 n+2}$

Now,

$$
p_{c q b}\left(F a_{2 n}, F a_{2 n+1}\right) \leq \lambda p_{c q b}\left(a_{2 n}, a_{2 n+1}\right)+
$$




$$
\begin{gathered}
\mu \frac{p_{c q b}\left(a_{2 n}, F a_{2 n}\right) p_{c q b}\left(a_{2 n+1}, F a_{2 n}\right)}{1+p_{c q b}\left(a_{2 n}, a_{2 n+1}\right)}+ \\
\delta \frac{\left[p_{c q b}\left(F a_{2 n}, a_{2 n+1}\right)+p_{c q b}\left(a_{2 n}, a_{2 n+1}\right)\right]\left[p_{c q b}\left(a_{2 n}, F a_{2 n+1}\right)\right]}{p_{c q b}\left(a_{2 n}, a_{2 n+1}\right)} \\
=\lambda p_{c q b}\left(a_{2 n}, a_{2 n+1}\right)+\delta \frac{p_{c q b}\left(a_{2 n}, a_{2 n+1}\right) p_{c q b}\left(a_{2 n}, a_{2 n+2}\right)}{p_{c q b}\left(a_{2 n}, a_{2 n+1}\right)} \\
=\lambda p_{c q b}\left(a_{2 n}, a_{2 n+1}\right)+\delta p_{c q b}\left(a_{2 n}, a_{2 n+2}\right) \\
\leq \lambda p_{c q b}\left(a_{2 n}, a_{2 n+1}\right)+\delta\left[p_{c q b}\left(a_{2 n}, a_{2 n+1}\right)+\right. \\
\left.p_{c q b}\left(a_{2 n+1}, a_{2 n+2}\right)\right] \text { So that, } \\
\left|p_{c q b}\left(a_{2 n+1}, a_{2 n+2}\right)\right| \leq \frac{\lambda+\delta}{1-\delta}\left|p_{c q b}\left(a_{2 n}, a_{2 n+1}\right)\right|
\end{gathered}
$$

Similarly let us take, $F a_{2 n+1}=a_{2 n+2}$ and $F a_{2 n+2}=a_{2 n+3}$ we obtain that,

$$
\left|p_{c q b}\left(a_{2 n+2}, a_{2 n+3}\right)\right| \leq \frac{\lambda+\delta}{1-\delta}\left|p_{c q b}\left(a_{2 n+1}, a_{2 n+2}\right)\right|
$$

Letting $g=\frac{\lambda-\delta}{1-\delta}$, we have

$$
\begin{gathered}
\left|p_{c q b}\left(a_{2 n+1}, a_{2 n+2}\right)\right| \leq g\left|p_{c q b}\left(a_{2 n}, a_{2 n+1}\right)\right| \leq \\
g^{2}\left|p_{c q b}\left(a_{2 n-1}, a_{2 n+1}\right)\right| \leq \ldots \ldots \ldots \ldots . . \leq g^{l+1}\left|p_{c q b}\left(a_{0}, a_{1}\right)\right|
\end{gathered}
$$

Then, for any $\mathrm{k}>1$, we obtain

$$
\begin{gathered}
\left|p_{c q b}\left(a_{k}, a_{n}\right)\right| \leq\left|p_{c q b}\left(a_{n}, a_{n+1}\right)\right|+ \\
\left|p_{c q b}\left(a_{n+1}, a_{n+2}\right)\right|+\ldots+\left|p_{c q b}\left(a_{k-1}, a_{k}\right)\right| \\
\left|p_{c q b}\left(a_{k}, a_{n}\right)\right| \leq\left|\left[g^{n}+g^{n+1}+\ldots \ldots .+g^{k-1}\right]\right| p_{c q b}\left(a_{0}, a_{1}\right) \mid \\
\left|p_{c q b}\left(a_{k}, a_{n}\right)\right| \leq \frac{g^{l}}{1-g}\left|p_{c q b}\left(a_{0}, a_{1}\right)\right| \\
\left|p_{c q b}\left(a_{k}, a_{n}\right)\right| \rightarrow 0 \text { as } k \rightarrow \infty
\end{gathered}
$$

Hence, $\left\{a_{k}\right\}$ represents Cauchy sequence. Since L is complete, there exists $h \varepsilon L$ such that

$$
a_{k} \rightarrow h \text { as } k \rightarrow \infty
$$

Since $F$ is continuous this implies that $F h=\lim _{k \rightarrow \infty} F a_{k}=\lim _{k \rightarrow \infty} a_{k+1}=h$

Then $h$ is fixed point of $F$.

Next to prove that $F$ has a unique fixed point.

On the contrary, Let us take $\mathrm{h}$ and $\mathrm{g}$ are different fixed points of F. Replace a by $\mathrm{h}$ and $\mathrm{b}$ by $\mathrm{g}$ in the (1), $p_{c q b}(h, g) \neq 0$.

Now,

$$
p_{c q b}(h, g)=p_{c q b}(F h, F g) \leq \lambda p_{c q b}(h, g)+
$$

$$
\begin{gathered}
\mu \frac{p_{c q b}(h, F h) p_{c q b}(g, F h)}{1+p_{c q b}(h, g)}+ \\
\delta \frac{\left[p_{c q b}(F h, g)+p_{c q b}(h, g)\right]\left[p_{c q b}(h, F g)\right]}{p_{c q b}(h, g)} \\
=\lambda p_{c q b}(h, g)+\delta\left[p_{c q b}(h, g)+p_{c q b}(h, g)\right] \\
=(\lambda+2 \delta) p_{c q b}(h, g)
\end{gathered}
$$

Therefore, we obtain $\left|p_{c q b}(h, g)\right|<\left|p_{c q b}(h, g)\right|$ which is a contradiction since $\lambda+2 \delta<1$.

Hence, $\mathrm{F}$ has a unique fixed point.

\section{Remark 2.1.}

Every complex metric space is a complex quasi metric space but the complex quasi metric space is not a complex metric space for example consider $L=[0,1]$. Define the function $p_{c q b}: L \times L \rightarrow C$ by $p_{c q b}(a, b)=4 i(a-b)$ for each $a, b \varepsilon L$. Clearly, $\left(L, p_{c q b}\right)$ is complex quasi metric space but it is not a complex valued metric space.

\section{Example 2.2.}

Let $L=[0,1]$. Define the function $p_{c q b}: L \times L \rightarrow C$ by $p_{c q b}(a, b)=i(a-b)$ for each $a, b \varepsilon L$. Clearly $\left(L, p_{c q b}\right)$ is complex quasi metric space. Consider a mapping $F: L \rightarrow L$ as $F(a, b)=\frac{(a+b)}{4}$ for each $a, b \varepsilon L$. Then, it can be show that the map $\mathrm{F}$ satisfies the condition of the Theorem 2.1 with positive real numbers $\lambda, \mu, \delta$. Hence, 0 is a unique fixed point of $\mathrm{F}$.

\section{Theorem2.2.}

Let $\left(L, p_{c q b}\right)$ be a complex valued quasi b metric space and also complete. Consider the mapping $F: L \rightarrow L$ as a continuous map. There is positive real numbers $\delta, \mu, \lambda$ with $\delta+\lambda+\mu<1 \quad p_{c q b}(F a, F b) \leq$ $\delta \mathrm{p}_{c q b}(a, b)+$

$$
\lambda \frac{\left[\mathrm{p}_{c q b}(a, F a)\right]^{2}\left[\mathrm{p}_{c q b}(a, F b)+\mathrm{p}_{c q b}(b, F a)\right]}{\left[1+\mathrm{p}_{c q b}(a, b)\right]^{2}}+
$$

$\mu \frac{\left[\mathrm{p}_{c q b}(F a, b)\right]^{2}\left[1+\mathrm{p}_{c q b}(b, F b)\right]}{\left[1+\mathrm{p}_{c q b}(a, b)\right]}$

for each $a, b \in L$. Then $\mathrm{F}$ has a fixed point.

\section{Proof.}

Define $F a_{n}=a_{n+1}, F a_{n+1}=a_{n+2}$

Suppose, 
$p_{c q b}\left(F a_{n}, F a_{n+1}\right)$

$\leq \delta p_{c q b}\left(a_{n}, a_{n+1}\right)$

$+\lambda \frac{\left[p_{c q b}\left(a_{n}, F a_{n}\right)\right]^{2}\left[p_{c q b}\left(a_{n}, F a_{n+1}\right)+p_{c q b}\left(a_{n+1}, F a_{n}\right)\right]}{\left[1+p_{c q b}\left(a_{n}, a_{n+1}\right)\right]^{2}}$

$+\mu \frac{\left[p_{c q b}\left(F a_{n}, a_{n+1}\right)\right]^{2}\left[1+p_{c q b}\left(a_{n+1}, F a_{n+1}\right)\right]}{1+p_{c q b}\left(a_{n}, a_{n+1}\right)}$

$$
=\delta \mathrm{p}_{c q b}\left(a_{n}, a_{n+1}\right)+
$$

$\lambda \frac{\left[\mathrm{p}_{c q b}\left(a_{n}, a_{n+1}\right)\right]^{2}\left[\mathrm{p}_{c q b}\left(a_{n}, a_{n+2}\right)+\mathrm{p}_{c q b}\left(a_{n+1}, a_{n+1}\right)\right]}{\left[1+\mathrm{p}_{c q b}\left(a_{n}, a_{n+1}\right)\right]^{2}}+$

$\mu \frac{\left[\mathrm{p}_{c q b}\left(a_{n+1}, a_{n+1}\right)\right]^{2}\left[1+\mathrm{p}_{c q b}\left(a_{n+1}, a_{n+2}\right)\right]}{1+\mathrm{p}_{c q b}\left(a_{n}, a_{n+1}\right)}$

$$
=\delta \mathrm{p}_{c q b}\left(a_{n}, a_{n+1}\right)+
$$

$$
\lambda \frac{\left[\mathrm{p}_{c q b}\left(a_{n}, a_{n+1}\right)\right]^{2}\left[\mathrm{p}_{c q b}\left(a_{n}, a_{n+2}\right)\right]}{\left[1+\mathrm{p}_{c q b}\left(a_{n}, a_{n+1}\right)\right]^{2}}
$$

Since, $\left|1+\mathrm{p}_{c q b}\left(a_{n}, a_{n+1}\right)\right|^{2}>\left|\mathrm{p}_{c q b}\left(a_{n}, a_{n+1}\right)\right|^{2}$

So that, $\left|p_{c q b}\left(a_{n+1}, a_{n+2}\right)\right| \leq \delta\left|p_{c q b}\left(a_{n}, a_{n+1}\right)\right|+$ $\left|p_{c q b}\left(a_{n}, a_{n+2}\right)\right| \leq \delta\left|p_{c q b}\left(a_{n}, a_{n+1}\right)\right|+$

$$
\lambda\left|\mathrm{p}_{c q b}\left(a_{n}, a_{n+1}\right)+\mathrm{p}_{c q b}\left(a_{n+1}, a_{n+2}\right)\right|
$$

$\left|\mathrm{p}_{c q b}\left(a_{n+1}, a_{n+2}\right)\right| \leq \frac{\delta+\lambda}{1-\lambda}\left|\mathrm{p}_{c q b}\left(a_{n}, a_{n+1}\right)\right|$ Let us

$$
\text { take } h=\frac{\delta+\lambda}{1-\lambda}
$$

Now,

$$
\begin{aligned}
& \left|\mathrm{p}_{c q b}\left(a_{n+1}, a_{n+2}\right)\right| \leq h\left|\mathrm{p}_{c q b}\left(a_{n}, a_{n+1}\right)\right| \leq \\
& h^{2}\left|\mathrm{p}_{c q b}\left(a_{n-1}, a_{n}\right)\right| \leq \ldots . . \leq h^{n+1}\left|\mathrm{p}_{c q b}\left(a_{0}, a_{1}\right)\right|
\end{aligned}
$$

Therefore for all $m>n$ for each $m, n \in N$

$$
\begin{gathered}
\left|\mathrm{p}_{c q b}\left(a_{n}, a_{m}\right)\right| \leq s\left[\left|\mathrm{p}_{c q b}\left(a_{n}, a_{n+1}\right)\right|+\left|\mathrm{p}_{c q b}\left(a_{n+1}, a_{m}\right)\right|\right] \\
\left|\mathrm{p}_{c q b}\left(a_{n}, a_{m}\right)\right| \leq s\left|\mathrm{p}_{c q b}\left(a_{n}, a_{n+1}\right)\right|+ \\
s^{2}\left[\left|\mathrm{p}_{c q b}\left(a_{n+1}, a_{n+2}\right)\right|+\left|\mathrm{p}_{c q b}\left(a_{n+2}, a_{m}\right)\right|\right]
\end{gathered}
$$

$$
\begin{gathered}
\left|\mathrm{p}_{c q b}\left(a_{n}, a_{m}\right)\right| \leq s\left|\mathrm{p}_{c q b}\left(a_{n}, a_{n+1}\right)\right|+\ldots \ldots \ldots . . \\
s^{m-n}\left|\mathrm{p}_{c q b}\left(a_{m-1}, a_{m}\right)\right|
\end{gathered}
$$

Therefore, we obtain that

$$
\begin{gathered}
\left|\mathrm{p}_{c q b}\left(a_{n}, a_{m}\right)\right| \leq s h^{n}\left|\mathrm{p}_{c q b}\left(a_{0}, a_{1}\right)\right|+\ldots \ldots \ldots . .+ \\
s^{m-n} h^{m-1}\left|\mathrm{p}_{c q b}\left(a_{0}, a_{1}\right)\right| \\
=\sum_{i=1}^{m-n} s^{i} h^{i+n-1}\left|\mathrm{p}_{c q b}\left(a_{0}, a_{1}\right)\right| \\
\left|\mathrm{p}_{c q b}\left(a_{n}, a_{m}\right)\right| \leq \sum_{i=1}^{m-n} s^{i} h^{i+n-1}\left|\mathrm{p}_{c q b}\left(a_{0}, a_{1}\right)\right| \\
=\sum_{t=n}^{m-1} s^{t} h^{t}\left|\mathrm{p}_{c q b}\left(a_{0}, a_{1}\right)\right| \\
\leq \sum_{t=n}^{m-1}(s h)^{t}\left|\mathrm{p}_{c q b}\left(a_{0}, a_{1}\right)\right|
\end{gathered}
$$

Hence, we obtain

$$
\begin{gathered}
\left|\mathrm{p}_{c q b}\left(a_{n}, a_{m}\right)\right| \leq \frac{(s h)^{n}}{1-s h}\left|\mathrm{p}_{c q b}\left(a_{0}, a_{1}\right)\right| \rightarrow 0 \\
\text { as } n, m \rightarrow \infty
\end{gathered}
$$

Hence, $\left\{a_{n}\right\}$ is a Cauchy sequence in L. Let $\left(L, p_{c q b}\right)$ be complete so there is $l \in L$ such that $a_{n} \rightarrow l$

Suppose $\mathrm{F}$ is continuous,

Therefore

$$
F l=\lim _{n \rightarrow \infty} F a_{n}=\lim _{n \rightarrow \infty} a_{n+1}=l .
$$

Thus, 1 has a fixed point of $F$.

Consider the theorem 2.2 without continuity condition on F, we obtain that

\section{Theorem2.3.}

Let $\left(L, p_{c q b}\right)$ be a complex valued quasi $\mathrm{b}$ metric space and also complete. Consider the mapping $F: L \rightarrow L$. There is positive real numbers $\delta, \mu, \lambda$ with $\delta+\lambda+\mu<1 \quad p_{c q b}(F a, F b) \leq \delta \mathrm{p}_{c q b}(a, b)+$ $\lambda \frac{\left[\mathrm{p}_{c q b}(a, F a)\right]^{2}\left[\mathrm{p}_{c q b}(a, F b)+\mathrm{p}_{c q b}(b, F a)\right]}{\left[1+\mathrm{p}_{c q b}(a, b)\right]^{2}}+$ $\mu \frac{\left[\mathrm{p}_{c q b}(F a, b)\right]^{2}\left[1+\mathrm{p}_{c q b}(b, F b)\right]}{\left[1+\mathrm{p}_{c q b}(a, b)\right]}$ for each $a, b \in L$. Then $\mathrm{F}$ has a fixed point.

\section{Proof.}

Similar proof can obtain of previous theorem.

To show that only 1 is a fixed point of $F$.

If not, 1 is not a fixed point of $F$ then $p_{c q b}(l, F l)=z>0, z \in C$

Let us now, 


$$
\begin{gathered}
z \leq s p_{c q b}\left(l, a_{n+2}\right)+s p_{c q b}\left(a_{n+2}, F l\right) \\
=s p_{c q b}\left(l, a_{n+2}\right)+s p_{c q b}\left(F a_{n+1}, F l\right) \\
\leq s p_{c q b}\left(l, a_{n+2}\right)+s\left[\delta \mathrm{p}_{c q b}\left(a_{n+1}, l\right)+\right. \\
\lambda \frac{\left[\mathrm{p}_{c q b}\left(a_{n+1}, F a_{n+1}\right)\right]^{2}\left[\mathrm{p}_{c q b}\left(a_{n+1}, F l\right)+\mathrm{p}_{c q b}(l, F l)\right]}{\left[1+\mathrm{p}_{c q b}\left(a_{n+1}, l\right)\right]^{2}}+ \\
\mu \frac{\left[\mathrm{p}_{c q b}\left(F a_{n+1}, l\right)\right]^{2}\left[1+\mathrm{p}_{c q b}(l, F l)\right]}{1+\mathrm{p}_{c q b}\left(a_{n+1}, l\right)}
\end{gathered}
$$

So that $z \leq 0$ as $n \rightarrow \infty$ in the above inequality which is impossible.

Thus, 1 is a fixed point of $F$.

In this theorem with or without continuity, a fixed point uniquely exists.

\section{Theorem 2.4.}

Let $\left(L, p_{c q b}\right)$ be a complex valued quasi b metric space and also complete. Consider the mapping $F: L \rightarrow L$. There is positive real numbers $\delta, \mu, \lambda$ with $\delta+\lambda+\mu<1 \quad p_{c q b}(F a, F b) \leq \delta \mathrm{p}_{c q b}(a, b)+$ $\lambda \frac{\left[\mathrm{p}_{c q b}(a, F a)\right]^{2}\left[\mathrm{p}_{c q b}(a, F b)+\mathrm{p}_{c q b}(b, F a)\right]}{\left[1+\mathrm{p}_{c q b}(a, b)\right]^{2}}+$ $\mu \frac{\left[\mathrm{p}_{c q b}(F a, b)\right]^{2}\left[1+\mathrm{p}_{c q b}(b, F b)\right]}{\left[1+\mathrm{p}_{c q b}(a, b)\right]}$

for each $a, b \in L$. Then $\mathrm{F}$ has a unique fixed point.

\section{Proof.}

From previous theorems either 2.2 or 2.3 we obtain $F$ has a fixed point $\&$ to set $\mathrm{F}$ is no-empty.

Suppose, let us consider $\mathrm{F}$ has two distinct fixed points that are $F u=u$ and $F l=l, u \neq l$

Now,

$$
\begin{gathered}
p_{c q b}(F l, F u) \leq \delta \mathrm{p}_{c q b}(l, u)+ \\
\lambda \frac{\left[\mathrm{p}_{c q b}(l, F l)\right]^{2}\left[\mathrm{p}_{c q b}(l, F u)+\mathrm{p}_{c q b}(u, F l)\right]}{\left[1+\mathrm{p}_{c q b}(l, u)\right]^{2}}+ \\
\mu \frac{\left[\mathrm{p}_{c q b}(F l, u)\right]^{2}\left[1+\mathrm{p}_{c q b}(u, F u)\right]}{\left[1+\mathrm{p}_{c q b}(l, u)\right]} \\
=\delta \mathrm{p}_{c q b}(l, u)+ \\
\lambda \frac{\left[\mathrm{p}_{c q b}(l, l)\right]^{2}\left[\mathrm{p}_{c q b}(l, u)+\mathrm{p}_{c q b}(u, l)\right]}{\left[1+\mathrm{p}_{c q b}(l, u)\right]^{2}}+ \\
\mu \frac{\left[\mathrm{p}_{c q b}(l, u)\right]^{2}\left[1+\mathrm{p}_{c q b}(u, u)\right]}{\left[1+\mathrm{p}_{c q b}(l, u)\right]}
\end{gathered}
$$

$$
\begin{gathered}
\left|p_{c q b}(l, u)\right| \leq(\delta+\mu)\left|\mathrm{p}_{c q b}(l, u)\right| \\
\left|p_{c q b}(l, u)\right|<\left|\mathrm{p}_{c q b}(l, u)\right|
\end{gathered}
$$

Since $\delta+\mu<1$ which is contradiction.

Hence, $1=u$. Therefore, $\mathrm{F}$ has a unique fixed point.

\section{Remark 2.3.}

Each complex valued b-metric space is a complex valued $b$-quasi metric space but the converse needs not be true.

For example, consider $L=[0,1]$. Consider a quasi b-metric space $p_{c q b}: L \times L \rightarrow C$

defined as

$$
p_{c q b}(g, f)=\frac{1}{2}[(g-f)+i(g-f)] \forall g, f \in L
$$

is a complex quasi b-metric space with $\mathrm{S}=2$ but converse part needs not be true .

\section{Example 2.4.}

Let $\mathrm{L}=[0,1]$. Consider a quasi b-metric space $\mathrm{p}_{\mathrm{cqb}}$ : $\mathrm{L} \mathrm{x}$ $\mathrm{L} \rightarrow \mathrm{C}$ defined as

$$
p_{c q b}(g, f)=\frac{3}{4}[(g-f)+i(g-f)] \forall g, f \in L
$$

It can easily verify that $\left(\mathrm{L}, \mathrm{p}_{\mathrm{cqb}}\right)$ is a complex quasi $\mathrm{b}$ metric space with $\mathrm{s}=3$. Let $\mathrm{F}: \mathrm{L} \rightarrow \mathrm{L}$ as $\mathrm{Fa}=\mathrm{a} / 3, \mathrm{Fb}=\mathrm{b} / 3$ for every $\mathrm{a}, \mathrm{b} \varepsilon \mathrm{L}$. Then, we can easily verify, the map $\mathrm{F}$ satisfies theorem 2.1 condition with positive real numbers,

$$
\lambda, \delta, \mu \& \delta+\mu<1 .
$$

Thus, unique fixed point is 0 of $F$.

\section{Conclusions}

In complex valued quasi $\mathrm{b}$-metric spaces, we proved the fixed point theorem by using rational contractive condition. A symmetric condition that does not exist in complex quasi $b$ metric spaces that are valued. Whereas the symmetric condition is present in complex valued metric spaces. In every other generalization of metric spaces, this sort of metric space generalization is not possible. Each complex b-metric space that is valued is a complex b-quasi-metric space that is valued, but the reverse needs not be valid.

\section{Conflict of Interest}

The authors note that there is no conflict of interest. 


\section{Acknowledgments}

For their comments and helpful feedback, the writers are sincerely thankful to the referees.

\section{REFERENCES}

[1] A. Azam, B, Fisher, M. Khan, Common fixed point theorems in complex valued metric spaces, Num. Func. Anal. Opt. 32, pp. 243-253, 2011

[2] S. Czerwik, Contraction mappings in b- metric spaces, Acta Mathematica et Informatica Universitatis Ostraviensis vol.1 No.1, 5-11, 1993

[3] K. Rao, P. Swamy and J. Prasad, A common fixed point theorem in complex valued b- metric spaces, Bulletin of Mathematics and Statistics Research, vol.1, no.1 2013.

[4] M. H. Shah and N. Hussian, Nonlinear contractions in partially ordered quasi b-metric spaces, Commun. Korean Math. Soc. 27(1) pp.117-128, 2012.

[5] S. Banach, "sur les operations dans les ensembles abstraits et leur application aux equation integrals", Fundamenta Mathematicae, vol.3, pp. 133-181, 1922.
[6] P. Dhivya and M. Marudai, Common fixed point theorems for mappings satisfying a contractive condition of rational expression on a ordered complex partial metric spaces, Cogent Mathematics 4:1389622, 2017.

[7] M. Imdad, T. I. Khan, On Common fixed point of pair wise coincidentally commuting non-continuous mappings satisfying a rational inequality, Bull. Calcutta Math. Soc. pp. 263-268, 2001.

[8] J. Uma Maheswari and A Anbarasan, Fixed points theorems of $(\kappa, \mu)$ rational contractive mappings in ordered complex valued quasi metric spaces, no.5, pp. 2791-2800, 2020

[9] F. Rouzkard and M. Imdad Some common fixed point theorems on complex valued metric spaces, Computers and Mathematics with Applications, Vol.64, no.6, pp. 1866-1874, 2012.

[10] Abbas, M, Cajbaic Rajic V, Nazir. T and Radenovic. "Common fixed point of mappings satisfying rational inequalities in ordered complex valued generalized metric spaces ", Afrika Matematika DOI 10.1007/s13370-013-018 $5-\mathrm{z}, 2013$.

[11] A.k Dubey, Rita Shukla and R.P. Dubey "Some fixed point theorems in complex valued b-metric spaces",Journal of complex systems. Vol.2015 Article ID832467, 7 pages, 2014 . 